\title{
Single-Dose Intraoperative Steroid Administration Does Not Impact Early Atrial Fibrillation Recurrence
}

Kolade M. Agboola

Mayo Clinic Rochester https://orcid.org/0000-0001-9253-738X

Michael Dietrich

Mayo Clinic Rochester

Roshan Karki

Mayo Clinic Rochester

Fahad Lodhi

Mayo Clinic Rochester

Trevon McGill

Mayo Clinic Rochester

Samuel J. Asirvatham

Mayo Clinic Rochester

Abhishek J. Deshmukh

Mayo Clinic Rochester

Christopher V. DeSimone ( $\square$ DeSimone.Christopher@mayo.edu )

Mayo Clinic Rochester

\section{Research Article}

Keywords: Atrial fibrillation, ablation, steroids, outcomes, recurrence

Posted Date: July 26th, 2021

DOI: https://doi.org/10.21203/rs.3.rs-672135/v1

License: (c) (i) This work is licensed under a Creative Commons Attribution 4.0 International License.

Read Full License 


\section{Abstract}

Purpose: To evaluate the effect of single-dose intravenous dexamethasone on AF recurrence following radiofrequency catheter ablation.

Methods: A cohort of 94 adult patients ( $>18$ years) underwent catheter ablation at Mayo Clinic Rochester from January to March 2019. Only first-time ablation patients were included, with all re-do ablations excluded to minimize heterogeneity. Administration of intraoperative dexamethasone $4 \mathrm{mg}$ or $8 \mathrm{mg}$ was determined by chart review from the procedure. At our institution, intraoperative intravenous steroids are administered for postoperative nausea and vomiting (PONV) prophylaxis at the discretion of the anesthesiologist. AF recurrence was determined by ECG or cardiac monitoring at less than 3 months or between 3 months and 1 year with an in-person follow-up visit.

Results: A total of $36.2 \%$ of patients received intravenous dexamethasone compared to $63.8 \%$ who did not (providing a 2:1 comparison group). The incidence of documented AF or flutter lasting greater than 30 seconds was $20.6 \%$ in the dexamethasone group versus $21.7 \%$ in the non-dexamethasone group, $p$ value 1.00. AF or atrial flutter recurrence from 3 months to 1 year was $20.6 \%$ in the dexamethasone group compared to $21.7 \%$ in the non-dexamethasone group, $\mathrm{p}$ value 1.00 .

Conclusion: These data suggest that intraoperative intravenous dexamethasone administered during AF ablation for postoperative nausea and vomiting prophylaxis does not have a significant effect on AF recurrence rates.

\section{Introduction}

Radiofrequency (RF) ablation is a widely utilized and highly effective treatment modality for symptomatic paroxysmal and persistent atrial fibrillation (AF). RF ablation for AF is known to increase serum biomarkers of inflammation, which may be due to acute myocardial injury and healing of ablation-related atrial lesions ${ }^{1}$. Individuals with early recurrence of AF within the first three months after AF ablation was shown to have significantly greater odds of having an increase in serum C-Reactive Protein (CRP) levels at post-ablation follow-up, suggesting an association between increased inflammation and early recurrence of $\mathrm{AF}^{2}$.

The effect of peri-procedural administration of nonsteroidal anti-inflammatory drugs and corticosteroids has been evaluated ${ }^{3-5}$. Peri-ablation administration of intravenous steroids followed by a short course of moderate-intensity oral steroids has been shown to significantly reduce AF recurrence at 3 months ${ }^{5}$. There is no evidence regarding the efficacy of intravenous dexamethasone, and it is unclear if single-dose administration at the time of ablation for the prevention of postoperative nausea (PONV) yields a similar reduction in $\mathrm{AF}$ recurrence or inhibits adequate lesions formation and maturation.

\section{Methods}


A cohort of 94 total adult patients was retrospectively selected in consecutive order from a chronological database of AF ablation patients. We included patients $>18$ years of age, undergoing catheter ablation with pulmonary vein isolation for paroxysmal and persistent AF between January and March of 2019. Only first-time ablation candidates were included and all re-do ablation candidates were excluded to minimize heterogeneity. Administration of intraoperative dexamethasone was determined by chart review of the procedural anesthesia records.

Intraoperative steroids have been shown to have efficacy in reducing PONV ${ }^{8-10}$. It is the practice within our institutions' anesthesiology group to administer prophylactic intravenous corticosteroids at the discretion of the anesthesiologist for PONV prophylaxis, independent of the electrophysiologist performing the ablation. Thus, at the time of the procedure, the operator was essentially "blinded" to the administration of dexamethasone. AF recurrence was determined by electrocardiogram or cardiac monitoring device at less than 3 months and 3 months to 1 year, which was obtained at the time of inperson follow-up visit. The protocol was approved by our institutional review board and all patients provided informed consent.

Demographic information for each patient was obtained via a review of the electronic medical record. Administration of dexamethasone and dosing was determined by review of procedural medication administration records. Dexamethasone dosing was determined at the discretion of the anesthesiologist. Patients in the dexamethasone group either received $4 \mathrm{mg}$ or $8 \mathrm{mg}$ of intravenous dexamethasone during the catheter ablation procedure. Patients in the control group did not receive any intravenous steroids including dexamethasone at the time of the catheter ablation procedure. Administration of other steroidal and non-steroidal anti-inflammatory agents at the time of ablation and in the postoperative period was also reviewed. Additionally, the concomitant use of antiarrhythmic agents was determined by chart review (Figure 1).

\section{Results}

A total of 94 patients were included in our study, from a population of 99 patients that were initially screened. The 5 patients that were excluded did not provide research study consent and thus were not included. Demographic data for the study population including age, gender, race, body mass index, and pertinent comorbidities are shown in Table 1. The mean age in our study population was $62.1 \pm 9.8$ years. The patients were predominantly male $(78.7 \%)$ and Caucasian (60.6\%). Our "treatment group" was comprised of 34 patients that received dexamethasone, and 60 patients in the control group (no dexamethasone).

The rates of AF and atrial flutter recurrence, defined as episodes lasting greater than 30 seconds, were not statistically different between the two groups. A total of 13 patients $(21.6 \%)$ in the control group and 7 patients (20.6\%) in the experimental group developed recurrence within the first 3 months, a p-value of 1.00. At 3 months to 1 year, 13 patients in the control group (21.6\%) and 7 patients (20.6\%) in the experimental group had developed recurrence. The rates of direct current cardioversion (DCCV) in these 
groups within the first 3 months were also not significantly different (Table 2). Within the control group, 8 out of 60 (13.3\%) patients required DCCV within the first 3 months compared to 4 out of $34(11.8 \%)$ patients in the experimental group, a p-value of 1.00. Similarly, at 3 months to 1 year, there was not a significant difference in the rates of DCCV between the two groups with 7 patients $(11.7 \%)$ in the control group and 3 patients $(8.8 \%)$ in the experimental group (Table 3$)$.

Within the control and experimental groups 38 out of 60 patients $(63.3 \%)$ and 20 out of 34 patients (58.8\%), respectively, were prescribed an antiarrhythmic agent within 3 months of their catheter ablation procedure. There was not a statistically significant difference in the administration of antiarrhythmic agents between these groups with a p-value of 0.67. At 3 months to 1 year, there was no statistically significant difference in the administration of antiarrhythmic agents between the control and experimental groups (Table 3). Rates of antiarrhythmic drug administration in the control and experimental groups were $35.6 \%$ (21 out of 60 patients) and $44.1 \%$ (15 out of 34 patients), respectively (pvalue 0.51$)$.

\section{Discussion}

In this study, we sought to evaluate the effect of single-dose intravenous dexamethasone on AF recurrence following RF catheter ablation. Patients in our study received $4 \mathrm{mg}$ or $8 \mathrm{mg}$ of dexamethasone at the time of AF ablation by anesthesia and were compared to controls which did not receive any intravenous steroids in a "blinded fashion" to the operator. Our main findings were that: 1) there was no significant difference in rates of documented AF recurrence, DCCV, or prescription of antiarrhythmic agents at 3 months or 1 year follow-up. These results were suggestive of the use of single-dose intravenous dexamethasone not being associated with early and late AF recurrence and thus of utility for PONV without affecting outcomes.

In a study of Paroxysmal AF patients, those randomized to colchicine experienced a significant reduction in early and late AF recurrence compared to those who received placebo ${ }^{3,4}$. The serum concentration of CRP and interleukin 6 were also significantly reduced after 4 days of treatment in the colchicine group, suggesting decreased systemic inflammation leading to reduced $A F$ recurrence ${ }^{3}$. Single dose intravenous corticosteroids administered at the time of ablation did not reduce post-ablation recurrence after $\mathrm{AF}^{6,7}$. Notably in both studies methylprednisolone and hydrocortisone were administered at the time of ablation $^{6,7}$.

The findings of our study are consistent with the findings of previous studies involving single-dose intravenous steroids at the time of catheter ablation. A prior study has shown that in 89 patients who received a single bolus injection of $100 \mathrm{mg}$ hydrocortisone within 30 minutes of completing the pulmonary vein isolation procedure, there was no significant difference in immediate, early, and late AF recurrence rates ${ }^{6}$. A similar result was found when comparing the effects of low-dose intravenous steroids with $100 \mathrm{mg}$ hydrocortisone and moderate-dose steroids with $125 \mathrm{mg}$ methylprednisolone in a prior study ${ }^{7}$. It was felt that moderate-dose steroids were thought to decrease post-ablation inflammation, 
evidenced by a significant reduction in maximum body temperature and serum C-reactive protein levels compared to the low-dose steroid and control groups. However, there was no significant difference in immediate, early, or midterm atrial fibrillation recurrence ${ }^{7}$.

Catheter ablation using radiofrequency energy involves the delivery of high frequency alternating electrical current, which heats the incident tissue underlying the catheter tip in a resistive or ohmic manner ${ }^{11}$. Following catheter ablation, there are both localized and systemic inflammatory responses, as well as a continued myocardial injury which results in the maturation of the newly formed lesions ${ }^{1}$. From a histological perspective, in vivo ablation studies in animal models have demonstrated infiltration of necrotic myocardium by lymphocytes and macrophages, ultimately resulting in the replacement of coagulative necrotic myocardium with fibrosis ${ }^{12}$. This post-ablation inflammatory response typically occurs within the first 3 days after ablation and is evidenced systemically by elevation in serum C-reactive protein (CRP) levels ${ }^{1}$.

AF recurrence following catheter ablation is thought to be in part mediated by systemic inflammation. Interestingly, patients without early recurrence of AF within the first month have higher CRP levels compared to individuals that experienced early recurrence ${ }^{13}$. This suggests that increased systemic inflammation may somehow be protective against early AF recurrence. One possible explanation for this is that the degree of systemic inflammation is indicative of or proportional to the degree of local inflammation at the site of myocardial lesions. As such, a heightened local inflammatory response may lead to a more robust lymphocytic and macrophagic infiltration, yielding more fibrosis and durable lesion formation. Interestingly, there was no difference in CRP levels between individuals with and without late recurrence, suggesting that systemic inflammation affects recurrence more acutely and less so chronically ${ }^{12}$.

The underlying mechanism of myocardial lesion formation following catheter ablation may provide insight into the potential effects of steroids and other anti-inflammatory agents. If steroids reduce the acute post-ablation inflammatory response that is responsible for lesion maturation, then steroids may impair lesion maturation and promote the late recurrence of AF. In our study, we did not observe such a phenomenon as there was not a significant difference in AF recurrence between individuals that received intraoperative dexamethasone and those that did not. As we have discussed, there is some evidence in support of the alternative hypothesis which suggests a benefit to suppression acute post-ablation inflammation with steroids and anti-inflammatory agents, such as colchicine ${ }^{3-5}$. However, these studies were performed with relatively small study populations and there has yet to be a large multicenter randomized trial to investigate this question. A more extensive and in-depth investigation is needed to determine the true effects of steroids on AF recurrence, as well as to determine the potential role of systemic and local inflammation in AF recurrence.

\section{Declarations}

Ethics approval and consent: Appropriate IRB approval was obtained for conducting this study. 
Consent for publication: Appropriate consent for publication as obtained.

Funding: No funding was utilized in the preparation of this manuscript.

Conflicts of interest/Competing interests: Drs. Agboola et al have no conflicts of interest or competing interests relevant to this manuscript.

Availability of data and material: Not applicable

Code availability: Not applicable

Author's contributions: All authors contributed to the study conception and design. Material preparation, data collection and analysis were performed by Drs. Kolade M. Agboola, Michael Dietrich, Roshan Karki, and Christopher V. DeSimone. The first draft of the manuscript was written by Dr. Kolade M. Agboola and all authors commented on previous versions of the manuscript. All authors read and approved the final manuscript.

Acknowledgements: All participating parties have been adequately acknowledged.

Compliance with Ethical Standards: We the authors have disclosed all potential conflicts of interest. Our research does involve human participants who have consented to have their medical data used for research purposes. Our research does not involve animal subjects.

\section{References}

1. Lim HS, Schultz C, Dang J, et al. Time course of inflammation, myocardial injury, and prothrombotic response after radiofrequency catheter ablation for atrial fibrillation. Circ Arrhythm Electrophysiol. Feb 2014;7(1):83-9. doi:10.1161/CIRCEP.113.000876

2. McCabe JM, Smith LM, Tseng ZH, et al. Protracted CRP elevation after atrial fibrillation ablation. Pacing Clin Electrophysiol. Sep 2008;31(9):1146-51. doi:10.1111/j.1540-8159.2008.01155.x

3. Deftereos S, Giannopoulos G, Kossyvakis C, et al. Colchicine for prevention of early atrial fibrillation recurrence after pulmonary vein isolation: a randomized controlled study. J Am Coll Cardiol. Oct 2012;60(18):1790-6. doi:10.1016/j.jacc.2012.07.031

4. Deftereos S, Giannopoulos G, Efremidis M, et al. Colchicine for prevention of atrial fibrillation recurrence after pulmonary vein isolation: mid-term efficacy and effect on quality of life. Heart Rhythm. Apr 2014;11(4):620-8. doi:10.1016/j.hrthm.2014.02.002

5. Kim YR, Nam GB, Han S, et al. Effect of Short-Term Steroid Therapy on Early Recurrence During the Blanking Period After Catheter Ablation of Atrial Fibrillation. Circ Arrhythm Electrophysiol. Dec 2015;8(6):1366-72. doi:10.1161/CIRCEP.115.002957

6. Won H, Kim JY, Shim J, et al. Effect of a single bolus injection of low-dose hydrocortisone for prevention of atrial fibrillation recurrence after radiofrequency catheter ablation. Circ J. 2013;77(1):53-9. doi:10.1253/circj.cj-12-0728 
7. Kim DR, Won H, Uhm JS, et al. Comparison of two different doses of single bolus steroid injection to prevent atrial fibrillation recurrence after radiofrequency catheter ablation. Yonsei Med J. Mar 2015;56(2):324-31. doi:10.3349/ymj.2015.56.2.324

8. De Oliveira GS, Castro-Alves LJ, Ahmad S, Kendall MC, McCarthy RJ. Dexamethasone to prevent postoperative nausea and vomiting: an updated meta-analysis of randomized controlled trials. Anesth Analg. Jan 2013;116(1):58-74. doi:10.1213/ANE.0b013e31826f0a0a

9. Apfel CC, Korttila K, Abdalla M, et al. A factorial trial of six interventions for the prevention of postoperative nausea and vomiting. N Engl J Med. Jun 2004;350(24):2441-51. doi:10.1056/NEJMoa032196

10. Carlisle J, Stevenson CA. WITHDRAWN: Drugs for preventing postoperative nausea and vomiting. Cochrane Database Syst Rev. 07 2017;7:CD004125. doi:10.1002/14651858.CD004125.pub3

11. Haines DE. The biophysics of radiofrequency catheter ablation in the heart: the importance of temperature monitoring. Pacing Clin Electrophysiol. Mar 1993;16(3 Pt 2):586-91. doi:10.1111/j.15408159.1993.tb01630.x

12. Nascimento T, Mota F, dos Santos LF, et al. Impact of prophylactic corticosteroids on systemic inflammation after extensive atrial ablation in pigs. Europace. Jan 2012;14(1):138-45. doi:10.1093/europace/eur259

13. Lellouche $N$, Sacher $F$, Wright $M$, et al. Usefulness of C-reactive protein in predicting early and late recurrences after atrial fibrillation ablation. Europace. May 2009;11(5):662-4. doi:10.1093/europace/eup077

\section{Tables}

Table 1. Population Demographics 


\section{Age at procedure (years)}

Mean (standard deviation)

$62.1(9.8)$

Median (Min, Max)

$64.5(29.0,79.0)$

\section{Gender}

Female

Male

Race

African American

American Indian/Alaskan Native

Asian Indian

Non-Hispanic or Latino

White

Did not identify

\section{BMI $\left(\mathrm{kg} / \mathrm{m}^{2}\right)$}

Mean (standard deviation)

Median (Min, Max)

\section{$\mathrm{CHA}_{2} \mathrm{DS}_{2}$ VASc Score}

Mean (standard deviation)

Median (Min, Max)

Diabetes Mellitus

No

Yes

Hypertension

No

Yes

\section{Heart Failure}

No

Yes
$20(21.3 \%$

$74(78.7 \%)$
1 (1.1\%)

1 (1.1\%)

$31(33.0 \%)$

57 (60.6\%)

$2(2.1 \%)$

$29.9(20.3,49.7)$

2.0 (1.3)

$2.0(0.0,7.0)$

$83(88.3 \%)$

$11(11.7 \%)$

$34(36.2 \%)$

60 (63.8\%)

$69(73.4 \%)$

25 (26.6\%) 


\begin{tabular}{|c|c|}
\hline Mean (standard deviation) & $55.7 \%(10.6)$ \\
\hline Median (Min, Max) & $59.0 \%(15.0,69.0)$ \\
\hline \multicolumn{2}{|l|}{ Diastolic Dysfunction } \\
\hline No & $47(50.0 \%)$ \\
\hline Yes & $47(50.0 \%)$ \\
\hline \multicolumn{2}{|l|}{ Left Atrial Volume Index } \\
\hline Mean (standard deviation) & $41.5(13.0)$ \\
\hline Median (Min, Max) & $40.5(16.0,78.0)$ \\
\hline \multicolumn{2}{|l|}{ Valvular Heart Disease } \\
\hline No & $85(91.4 \%)$ \\
\hline Yes & $8(8.6 \%)$ \\
\hline \multicolumn{2}{|l|}{ Stroke or TIA } \\
\hline No & $85(90.4 \%)$ \\
\hline Yes & $9(9.6 \%)$ \\
\hline \multicolumn{2}{|l|}{ Coronary Artery Disease } \\
\hline No & $76(80.9 \%)$ \\
\hline Yes & $18(19.1 \%)$ \\
\hline \multicolumn{2}{|l|}{ Obstructive Sleep Apnea } \\
\hline No & $48(51.1 \%)$ \\
\hline Yes & $46(43.6 \%)$ \\
\hline \multicolumn{2}{|l|}{ CPAP Therapy } \\
\hline No & $53(56.4 \%)$ \\
\hline Yes & $41(43.6 \%)$ \\
\hline \multicolumn{2}{|l|}{ Autoimmune Disease } \\
\hline No & $90(95.7 \%)$ \\
\hline Yes & $4(4.3 \%)$ \\
\hline \multicolumn{2}{|l|}{ Malignancy } \\
\hline No & 90 (95.7\%) \\
\hline
\end{tabular}




\begin{tabular}{|c|c|}
\hline Yes & $4(4.3 \%)$ \\
\hline \multicolumn{2}{|l|}{ Renal Function - Creatinine } \\
\hline Mean (standard deviation) & $1.1(0.2)$ \\
\hline Median (Min, Max) & $1.1(0.6,1.8)$ \\
\hline \multicolumn{2}{|l|}{ Hepatic Function - AST, ALT } \\
\hline Mean (SD) & $34.2(24.0), 33.9(35.0)$ \\
\hline Median (Min, Max) & $27.0(12.0,140.0), 29.5(8.0,319.0)$ \\
\hline \multicolumn{2}{|c|}{ Anti-inflammatory Medication } \\
\hline No & $42(44.7 \%)$ \\
\hline Yes & $52(55.3 \%)$ \\
\hline \multicolumn{2}{|c|}{ Intraoperative Dexamethasone } \\
\hline No & $60(63.8 \%)$ \\
\hline Yes & $34(36.2 \%)$ \\
\hline \multicolumn{2}{|c|}{ Antiarrhythmic Agent within 3 months } \\
\hline No & $36(38.3 \%)$ \\
\hline Yes & $58(61.7 \%)$ \\
\hline \multicolumn{2}{|l|}{ Antiarrhythmic Agent } \\
\hline Sotalol & $15(25.9 \%)$ \\
\hline Dofetilide & $8(13.8 \%)$ \\
\hline Amiodarone & $21(36.2 \%)$ \\
\hline Flecainide & $11(19.0 \%)$ \\
\hline Propafenone & $2(3.4 \%)$ \\
\hline Dronedarone & $1(1.7 \%)$ \\
\hline \multicolumn{2}{|c|}{ Antiarrhythmic Agent within 3 months to 1 year } \\
\hline No & $57(61.3 \%)$ \\
\hline Yes & $36(38.7 \%)$ \\
\hline \multicolumn{2}{|l|}{ Antiarrhythmic Agent } \\
\hline Sotalol & $11(32.4 \%)$ \\
\hline Dofetilide & $3(8.8 \%)$ \\
\hline
\end{tabular}


Amiodarone

Flecainide

Propafenone
$10(29.4 \%)$

9 (26.5\%)

$1(2.9 \%)$

Table 2:

\begin{tabular}{|llll|}
\hline & Control (N = 60) & Dexamethasone (N = 34) & P-value \\
\hline Antiarrhythmic use & & & \\
\hline No & $22(36.7 \%)$ & $14(41.2 \%)$ & 0.67 \\
\hline Yes & $38(63.3 \%)$ & $20(58.8 \%)$ & \\
\hline DCCV & & & 1.00 \\
\hline No & $52(86.7 \%)$ & $30(88.2 \%)$ & \\
\hline Yes & $8(13.3 \%)$ & $4(11.8 \%)$ & 1.00 \\
\hline AF Recurrence & & & \\
\hline No & $47(78.3 \%)$ & $27(79.4 \%)$ & \\
\hline Yes & $13(21.7 \%)$ & $7(20.7 \%)$ & \\
\hline
\end{tabular}

Table 3: 


\begin{tabular}{|c|c|c|c|}
\hline & Control $(N=60)$ & Dexamethasone $(\mathrm{N}=34)$ & P-value \\
\hline \multicolumn{4}{|c|}{ Antiarrhythmic use } \\
\hline No & $38(64.4 \%)$ & $19(55.9 \%)$ & 0.51 \\
\hline Yes & $21(35.6 \%)$ & $15(44.1 \%)$ & \\
\hline \multicolumn{4}{|c|}{ DCCV } \\
\hline No & $53(88.3 \%)$ & $31(91.2 \%)$ & 0.74 \\
\hline Yes & $7(11.7 \%)$ & $3(8.8 \%)$ & \\
\hline \multicolumn{4}{|c|}{ AF Recurrence } \\
\hline No & $47(78.3 \%)$ & $27(79.4 \%)$ & 1.00 \\
\hline Yes & $13(21.7 \%)$ & 7 (20.7\%) & \\
\hline
\end{tabular}

\section{Figures}

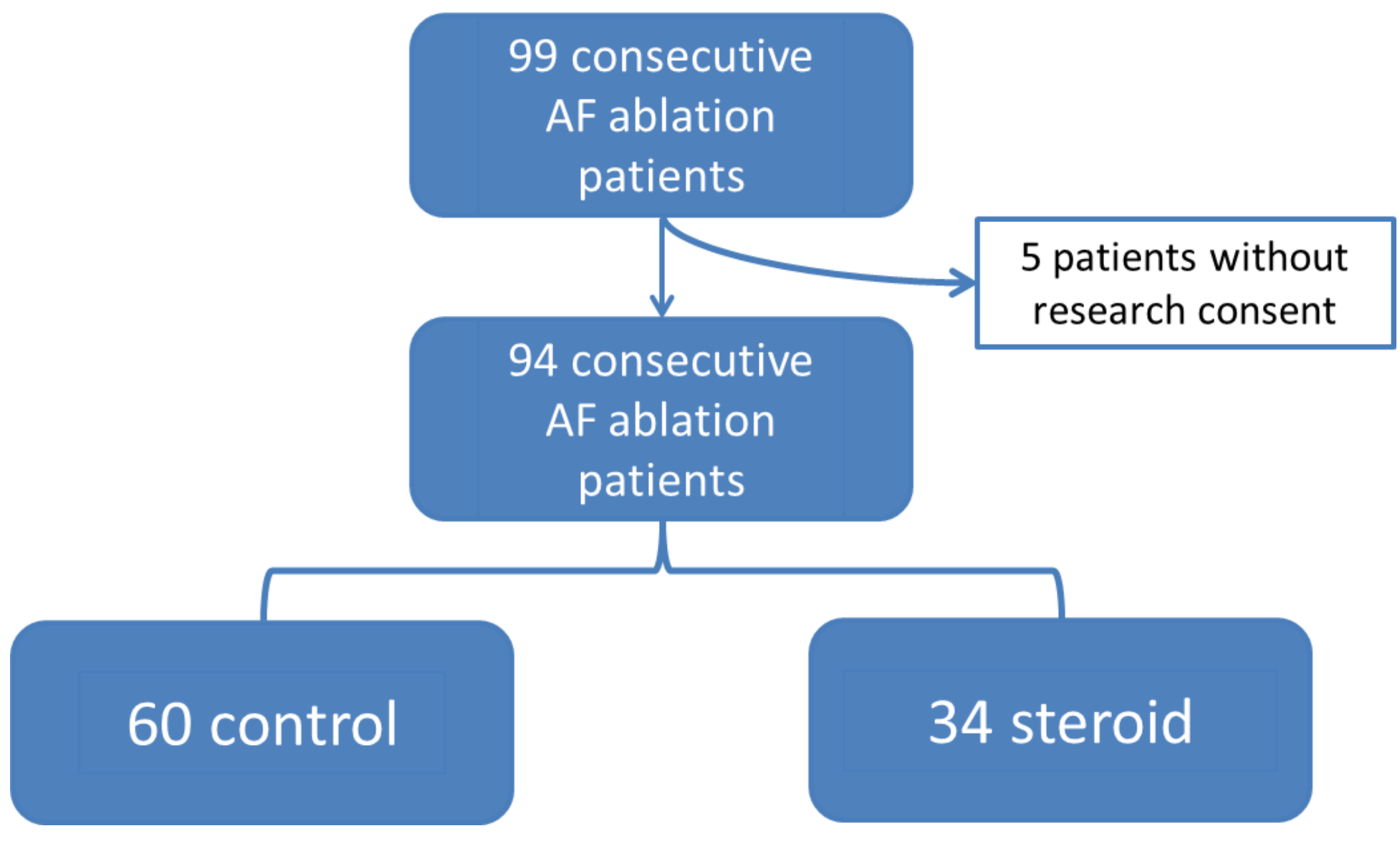

Figure 1 
Study Design Schematic

Page 13/13 